OPEN ACCESS

Edited by:

Benjamin Frey,

University Hospital Erlangen, Germany

Reviewed by:

Antonio Rozzi,

Centre Hospitalier Régional

Metz, France

Marlen Haderlein,

University of Erlangen

Nuremberg, Germany

*Correspondence:

Tongyu Lin

tongyulin@hotmail.com

${ }^{\dagger}$ These authors have contributed equally to this work

Specialty section

This article was submitted to Cancer Molecular Targets and

Therapeutics,

a section of the journal

Frontiers in Oncology

Received: 08 March 2020

Accepted: 21 May 2020

Published: 19 June 2020

Citation:

Zhang $M$, Huang $H$, Li X, Huang $Y$,

Chen C, Fang X, Wang Z, Guo C,

Lam S, Fu X, Hong H, Tian Y, LU T and

Lin T (2020) Long-Term Survival of

Patients With Chemotherapy-Naive

Metastatic Nasopharyngeal

Carcinoma Receiving Cetuximab Plus

Docetaxel and Cisplatin Regimen.

Front. Oncol. 10:1011.

doi: 10.3389/fonc.2020.01011

\section{Long-Term Survival of Patients With Chemotherapy-Naïve Metastatic Nasopharyngeal Carcinoma Receiving Cetuximab Plus Docetaxel and Cisplatin Regimen}

\author{
Mengping Zhang ${ }^{1,2 \dagger}$, He Huang ${ }^{1 \dagger}$, Xueying $\mathrm{Li}^{1,3 \dagger}$, Ying Huang ${ }^{4 \dagger}$, Chunyan Chen ${ }^{4}$, \\ Xiaojie Fang ${ }^{1}$, Zhao Wang ${ }^{1}$, Chengcheng Guo ${ }^{1}$, Sioteng $\mathrm{Lam}^{1,5}$, Xiaohong Fu ${ }^{1,6}$, \\ Huangming Hong ${ }^{1}$, Ying Tian ${ }^{1}$, Taixiang Lu ${ }^{4}$ and Tongyu Lin ${ }^{1 *}$
}

\begin{abstract}
'State Key Laboratory of Oncology in South China, Collaborative Innovation Center for Cancer Medicine, Department of Medical Oncology, Sun Yat-sen University Cancer Center, Guangzhou, China, ${ }^{2}$ Department of Oncology, The First Affiliated Hospital of Sun Yat-sen University, Guangzhou, China, ${ }^{3}$ Department of Medical Oncology, The Seventh Affiliated Hospital, Sun Yat-sen University, Shenzhen, China, ${ }^{4}$ State Key Laboratory of Oncology in South China, Collaborative Innovation Center for Cancer Medicine, Department of Radiation Oncology, Sun Yat-sen University Cancer Center, Guangzhou, China, ${ }^{5}$ Centro Hospitalar Conde de Sáo Januário, Macau, China, ${ }^{6}$ Department of Oncology, Shenzhen Nanshan Hospital, Shenzhen, China
\end{abstract}

Purpose: Metastatic nasopharyngeal carcinoma (mNPC) remains incurable. This prospective study aimed to investigate whether adding cetuximab to cisplatin-based induction therapy could improve efficacy and survival for chemotherapy-naïve mNPC patients.

Patients and Methods: Eligible chemotherapy-naïve mNPC patients were enrolled, including those initially diagnosed with $\mathrm{mNPC}(\mathrm{IM})$ and those with first-relapse metastases after radiotherapy (RM). Patients all received induction chemotherapy (IC) including docetaxel and cisplatin plus cetuximab. Those who obtained objective remission after IC would continue to receive radiotherapy concurrent with cetuximab and cisplatin, and further capecitabine as maintenance. Contemporaneous patients who received conventional therapy served as controls.

Results: Forty-three patients were enrolled, including $17 \mathrm{IM}$ and $26 \mathrm{RM}$ patients. Thirty-nine (90.7\%) patients had WHO III subtype. The overall response and complete response (CR) rates were, respectively, 79.1 and $34.9 \%$ after induction therapy and 76.7 and $46.5 \%$ after chemoradiotherapy. The 5-year overall survival (OS) and progression-free survival (PFS) rates reached 34.9 and 30\%, respectively, Subgroup analysis showed that compared with RM patients, IM patients had a higher 5-year OS (58.8 vs. 19.2\%) and PFS (52.9 vs. 19.2\%). The IM group had a higher CR rate of induction treatment than the RM group (52.9 vs. 23.1\%). No treatment-related death was observed. Twelve patients $(27.9 \%)$ remained alive with disease-free survival times from $60+$ to $135+$ months. Control patients showed a substantially lower survival rate (5-year OS, 10.9\%) and few long-term survivors. 
Conclusions: This regimen resulted in significantly improved efficacy and survival, which indicates a potentially curative role for chemotherapy-naïve mNPC, especially in newly diagnosed patients. A phase III clinical trial (NCT02633176) is ongoing for confirmation.

Keywords: survival, chemotherapy, metastatic nasopharyngeal carcinoma, cetuximab, induction therapy

\section{INTRODUCTION}

Nasopharyngeal carcinoma (NPC) is epidemic in southern China and Southeast Asia (1). Additionally, $\sim 25-30 \%$ of NPC patients exhibit metastatic disease (2), and 15\% of all NPC patients present with distant metastases at primary diagnosis (3). The outcomes of patients with metastatic NPC (mNPC) are heterogeneous, and long-term survival is possible in very few patients (4). On the basis of high-level evidence, patients with recurrent or primary $\mathrm{mNPC}$ generally have very poor survival, with a median overall survival of 11.5-15 months reported 10 years ago $(5,6)$ and a median survival of 29.1 months reported in 2016 (7). Generally, mNPC is recognized as an incurable disease, as few patients survive beyond 5 years.

Platinum-containing doublet regimens or concurrent chemoradiotherapy (CCRT) alone or induction chemotherapy followed by chemoradiotherapy continue to be regarded as standard first-line treatments for patients with recurrent or metastatic NPC. Gemcitabine, capecitabine, paclitaxel, and docetaxel have also been combined with cisplatin and yield similar survival $(8,9)$. However, no randomized trials have defined the optimum regimens.

Cetuximab is an IgG1 monoclonal antibody that inhibits ligand binding to the epidermal growth factor receptor (EGFR) (10). EGFR expression is reported in more than $85 \%$ of undifferentiated NPCs and is associated with a poor clinical outcome (11). Radiotherapy and platinum-based chemotherapy plus cetuximab have enhanced activity against head and neck cancer, with improved overall survival (OS) $(12,13)$. Although distinct differences exist between NPC and other head and neck cancers, despite originating from a similar cell or tissue lineage, we speculated that adding an EGFR inhibitor to platinumbased chemotherapy and CCRT could be beneficial for mNPC. Moreover, a phase 2 study of cetuximab in combination with a cytotoxic agent showed clinical activity and an acceptable safety profile in heavily pretreated patients with mNPC (14).

A meta-analysis of 11 randomized trials showed that longer first-line chemotherapy is associated with longer OS (15). However, prolongation of docetaxel or cisplatin exposure until disease progression is unrealistic because of cumulative toxic effects. Therefore, switching to a more tolerable chemotherapy, such as capecitabine, as a maintenance regimen might be a more effective treatment strategy.

We therefore conducted this single-center, prospective study of an epidermal growth factor receptor antibody (cetuximab)containing induction therapy and chemoradiotherapy regimen to investigate whether it would significantly improve survival outcomes while maintaining tolerability in mNPC patients without prior systemic therapy and would alter the therapeutic modality from conventional palliative to curative treatment.

\section{METHODS}

\section{Study Design and Patients}

We performed an investigator-initiated, open-label, single arm, single center, phase 2 trial at Sun Yat-sen University Cancer Center, Guangzhou, China. Eligible participants were 18 to 65 years of age and had histologically confirmed mNPC, including initial diagnosed NPC with metastases (IM) and first-relapse metastases after curative radiotherapy without neoadjuvant or adjuvant chemotherapy (RM). Pretreatment staging and metastases were confirmed via positron emission tomography/computerized tomography scans (PET/CT). Eligible patients had a type II or III histological subtype according to the WHO classification. Other eligibility criteria were as follows: patients had not received any previous systemic chemotherapy for recurrent or metastatic disease; had an Eastern Cooperative Oncology Group (ECOG) performance status of 0 or 1 ; had not received previous treatment with any investigational drug, surgery, irradiation or other anticancer therapies within the prior 4 weeks; had no known brain metastases; had adequate organ function as defined by adequate bone marrow function (hemoglobin $\geq 90 \mathrm{~g} / \mathrm{L}, \mathrm{WBC}$ count $\geq 3 \times 10^{9} / \mathrm{L}$, platelet count $\geq 100 \times 10^{9} / \mathrm{L}$ ), renal function (serum creatinine $\leq 140$ $\mu \mathrm{mol} / \mathrm{L}$ or calculated creatinine clearance $\geq 40 \mathrm{~mL} / \mathrm{min}$ ), and liver function (ALT or AST $\leq 3 \times$ the upper limit of normal, bilirubin $\leq 2 \times$ the upper limit of normal); had no uncontrolled cardiac or other disease with life expectancy of 3 months or more; provided written informed consent; and was amenable for regular follow-up. The study protocol was approved by the ethics committee of Sun Yat-sen University Cancer Center.

\section{Procedures}

The induction chemotherapy regimen was repeated every 3 weeks and comprised the following: intravenous docetaxel 75 $\mathrm{mg} / \mathrm{m}^{2}$ day 1 ; cisplatin at $25 \mathrm{mg} / \mathrm{m}^{2}$ on days 1,2 , and 3 ; and cetuximab at $250 \mathrm{mg} / \mathrm{m}^{2}$ on days 0,7 , and 14 with an initial dose of $400 \mathrm{mg} / \mathrm{m}^{2}$. This induction regimen was followed by CCRT consisting of intensity-modulated radiotherapy (IMRT) plus concomitant cetuximab $\left(250 \mathrm{mg} / \mathrm{m}^{2} /\right.$ week for 6 cycles $)$ and cisplatin ( $75 \mathrm{mg} / \mathrm{m}^{2} / 3$ weeks for 2 cycles). IMRT was given at $68-$ 70 Gy over 30 daily fractions over 6 weeks to the planning target volume of the existing primary tumor in IM patients, or 64$66 \mathrm{~Gy}$ in RM patients with previous radiotherapy, with additional radiotherapy of 62-66 Gy over 30 fractions to metastatic regional neck nodes if indicated. After CCRT, capecitabine was continued 
as maintenance therapy (cycles were repeated every 21 days with $1,000 \mathrm{mg} / \mathrm{m}^{2}$ twice daily, days 1 through 14 ).

Patients received this induction therapy regimen for a maximum of six cycles or until disease progression, death, intolerable toxicities, or patient request to stop. Furthermore, only patients who obtained complete or partial responses (CR or PR) after induction therapy could receive CCRT. For patients with locoregional metastatic bone lesions, additional radiotherapy with $30-40$ Gy in 10-20 fractions to these sites of lesions was performed. Patients with other residual metastatic foci in lung, liver, and non-cervical lymph nodes after induction therapy that was amenable to local therapy were offered surgery or radiofrequency ablation before CCRT. For patients who exhibited a CR after CCRT, maintenance therapy was continued for up to 3 years or until unacceptable toxicity, disease progression, or death.

Treatment-emergent adverse events (AEs) were assessed with the Common Terminology Criteria for Adverse Events version

TABLE 1 | Demographic and clinical characteristics.

\begin{tabular}{|c|c|}
\hline Characteristics & NO. (\%) \\
\hline NO. & 43 \\
\hline \multicolumn{2}{|l|}{ Gender } \\
\hline Female & $7(16.3)$ \\
\hline Male & $36(83.7$ \\
\hline \multicolumn{2}{|l|}{ Age, years ${ }^{\star}$} \\
\hline Median & 43 \\
\hline Range & $23-63$ \\
\hline \multicolumn{2}{|c|}{ ECOG performance status } \\
\hline 0 & $14(32.6$ \\
\hline 1 & $29(67.4$ \\
\hline \multicolumn{2}{|l|}{ Histology } \\
\hline WHO type 2 & $4(9.3)$ \\
\hline WHO type 3 & $39(90.7$ \\
\hline \multicolumn{2}{|l|}{ EBV-DNA status } \\
\hline Positive* $^{*}$ & $32(74.4$ \\
\hline Negative & $11(25.6$ \\
\hline \multicolumn{2}{|c|}{ Number of metastatic organs } \\
\hline 1 & $27(62.8$ \\
\hline 2 & $8(18.6)$ \\
\hline$\geq 3$ & $8(18.6)$ \\
\hline \multicolumn{2}{|c|}{ Sites of disease at registration } \\
\hline Distant lymph node & 7 (16.3) \\
\hline Bone & $32(74.4$ \\
\hline Liver & $14(32.6$ \\
\hline Lung & $11(25.6$ \\
\hline Others & $6(14.0)$ \\
\hline \multicolumn{2}{|l|}{ Prior radiotherapy } \\
\hline Yes & $26(60.5$ \\
\hline No & $17(39.5$ \\
\hline
\end{tabular}

Data are presented as a number
DNA copies $\geq 10^{3}$ copies $/ \mathrm{mL}$.
3.0 and were noted separately for the induction, CCRT, and maintenance treatment. The indications for cetuximab dose adjustment or interruption were described previously (14). The chemotherapy was continued independent of any temporary interruption of cetuximab. Cetuximab was not withheld for chemotherapy-related toxicities, unless the patient developed a concomitant illness that, in the opinion of the investigator, mandated interruption of therapy.

Tumor response was assessed by CT imaging according to RECIST version 1.1 by the independent image committee every two cycles during induction therapy and every 3 weeks during CCRT. CR and PR were defined, respectively, as $100 \%$ or at least $30 \%$ decrease in the sum of the longest diameters of target lesions compared with baseline. Follow-up was performed at the outpatient clinic every 1-3 months for the first year, every 3 months for the second year, every 6 months for the third to fifth years, and annually thereafter.

\section{Outcomes}

The primary objective was to determine progression-free survival (PFS), which was defined as the time from treatment initiation

TABLE 2 | Antitumor efficacy.

\begin{tabular}{llll}
\hline Variable $\quad \operatorname{IM}(n=17)$ & $\mathrm{RM}(n=26)$ & $\begin{array}{l}\text { Overall } \\
(n=43)\end{array}$
\end{tabular}

Response after induction chemotherapy, $\boldsymbol{n}(\%)$

Complete response

Partial response

Stable disease

Progressive disease

Overall response, \% [95\%

Cl])

Disease control

$\begin{array}{ccc}9(52.9) & 6(23.1) & 15(34.9) \\ 7(41.2) & 12(46.2) & 19(44.2) \\ 0(0) & 6(23.1) & 6(14.0) \\ 1(5.9) & 2(7.7) & 3(7.0) \\ 94.1 & 69.2 & 79.1 \\ {[82.9-100]} & {[51.5-87]} & {[66.9-91.2]} \\ 94.1 & 92.3 & 93[85.4-100] \\ {[82.9-100]} & {[82.1-100]} & \end{array}$

Response after

chemoradiotherapy,

n (\%)

Complete response

Partial response

Stable disease

Progressive disease

Overall survival

Median, months [95\% Cl]

Unreached*
$\begin{gathered}88.2 \\ 58.8\end{gathered}$

\section{8 (30.8)}

$20(46.5)$

$4(23.5)$

$9(34.6)$

$13(30.2)$

$0(0)$

$6(23.1)$

$6(14.0)$

$1(5.9)$

$3(11.5)$

$4(9.3)$

2-year rate, \%

5-year rate, \%

Progression-free survival

Median, months [95\% Cl]

Unreached* $^{*}$

2-year rate, \%

58.8

5-year rate, \%

52.9

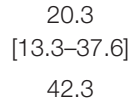

32.9

[18.2-47.5]

60.5

34.9

*Indicates that the IM subgroup significantly differed from RM subgroup; Cl, confidence interval. 
to disease progression or death from any cause, whichever came first. Secondary endpoints included the proportion of patients who had a confirmed overall response (OR) (defined as CR or PR lasting at least 4 weeks according to the RECIST 1.1), OS (defined as the time from treatment initiation to the date of death or last follow-up), and AEs. Patients were considered long-term survivors if they were disease-free for a period of more than 60 months without any treatment except maintenance treatment after a CR.

\section{Statistical Analyses}

The asymptotic distribution, provided in Lachin [(16), p. 409411] was used to calculate the sample size for this single arm trial. The justification for the sample size is explained below. The twosides Type I error rate was set at 5\%, and the type II error rate set at $20 \%$, giving $80 \%$ power. The accrual period was set at 1 year, and the total study period was set at 2 years. The OS rate at 1 year, based upon a previous study (17), is as high as $60 \%$ among patients treated with platinum-based therapy. Among patients receiving the novel regimen, the 1 -year OS rate was expected to increase to $80 \%$. This difference of $20 \%$ equates to a hazard ratio of 0.44 . The sample size calculation, given the above information, estimates that 12 events were needed. Finally, it was estimated that 25 patients were required to achieve this number of events allowing for a $10 \%$ loss to follow-up/non-adherence rate.

PFS and OS were estimated using the Kaplan-Meier method. Hazard ratios were calculated by the use of the Cox proportionalhazards model. The response rate and its 95\% CI (using the method of Pearson and Clopper) were calculated. We performed subgroup analyses among subgroups between mNPC patients with IM and RM for OS and PFS and response rate. We performed post-hoc subgroup analyses for OS and PFS, focusing on CR after induction therapy. We calculated the median followup time as the median of all enrolled patients, irrespective of whether the patients had died (18). Descriptive statistics were used for safety evaluations. All statistical testing was two-sided

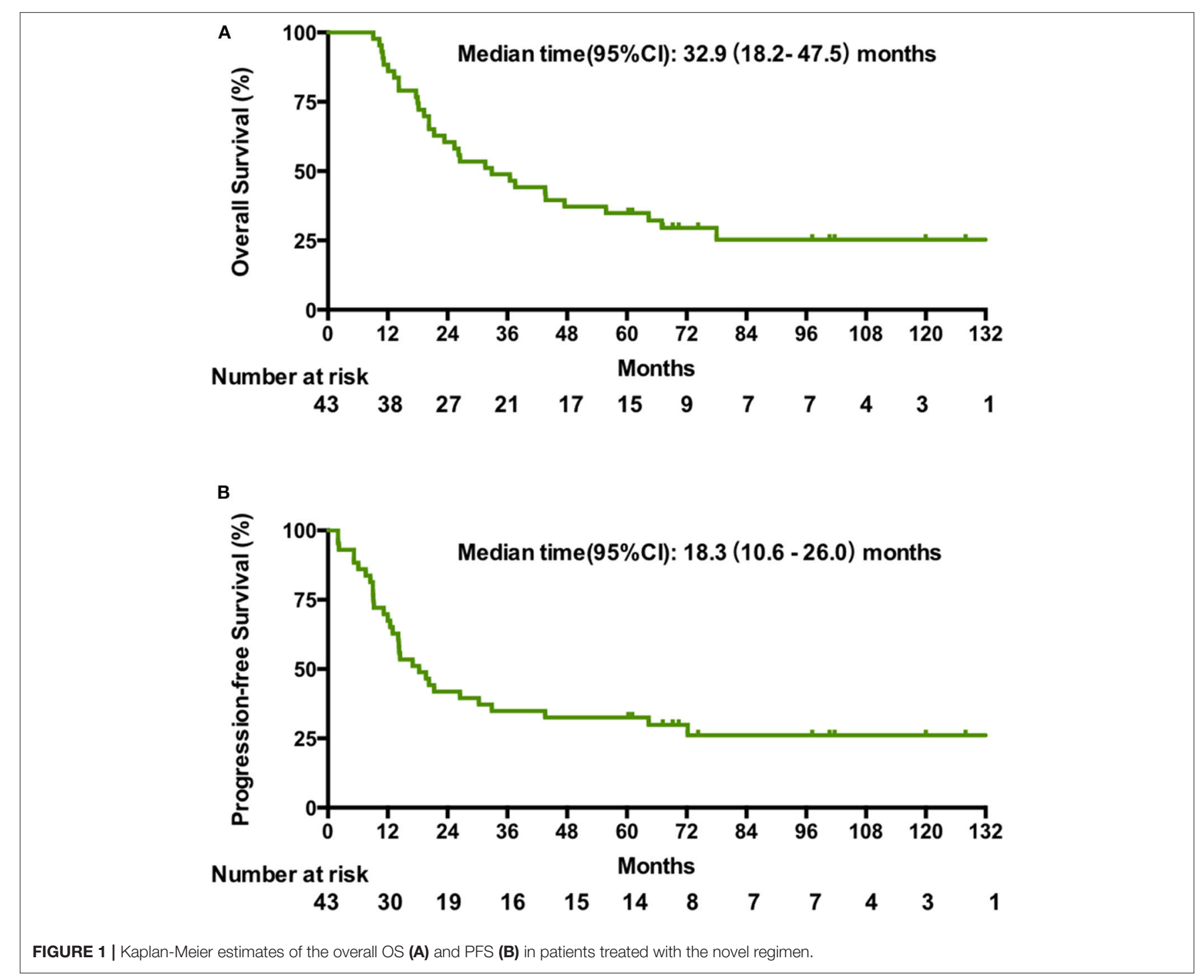


at the nominal 5\% significance level. All analyses were performed with SPSS 13.0.

\section{RESULTS}

Between July 2006 and December 2014, we enrolled 43 patients, $17(39.5 \%)$ with initial diagnosis of NPC with metastases (IM) and $26(60.5 \%)$ with first-relapse metastases (RM). All patients had evidence of EGFR-positive NPC. Table 1 summarizes the baseline characteristics of all 43 enrolled patients.

After the completion of induction chemotherapy, median cycles given to patients were 5 cycles (IQR 4-6). The OR rate was $79.1 \%$, and 15 of 43 patients (34.9\%) had a CR at all disease sites. Cetuximab was interrupted in 5 patients $(11.6 \%)$ due to grade 3 acneiform skin rash. Six (13.9\%) required a dose reduction of cisplatin or docetaxel during induction therapy due to serious myelosuppressive toxicity. Thirty-four patients obtained a CR or PR after induction chemotherapy, including $16 \mathrm{IM}$ patients and $18 \mathrm{RM}$ patients, and went on to receive CCRT; the OR and CR rates after CCRT were 76.7 and 46.5\%, respectively (Table 2). Due to drug-related toxicity or patient refusal, only 15 patients received capecitabine as maintenance following $\mathrm{CR}$ after CCRT, among which 5 patients had disease progression during this period.

The cutoff date for survival analysis was July 30, 2018. The median follow-up time for survival was 89 months (range, 32135). During follow-up, 31 patients had disease progression and finally died. After documented SD or PD during treatment or follow-up period, patients received second-line or third-line chemotherapy or palliative radiotherapy or did not receive any antitumor therapy. The median OS was 32.9 months (95\% CI, 18.2-47.5). Kaplan-Meier estimated OS rates at 6 months, 1, 2, 3 , and 5 years were $100,86,60.5,46.4$, and $34.9 \%$ respectively (Figure 1A). The median PFS was 18.3 months (95\% CI, 10.6-26 months). The PFS at 6 months, 1, 2, 3, and 5 years was $86,67.4$,
41.9, 34.9, and 30\% respectively (Figure 1B). Contemporaneous patients in the same hospital received conventional regimen showed poorer survival: for OS, median OS, $21 \mathrm{mo}, 95 \%$ CI, 17.8-24.0, HR = 2.1, 95\% CI, 1.3-3.3; for PFS, median PFS, 8 mo, 95\% CI, 6.4-9.6 mo, HR = 3.3, 95\% CI, $2.1-5.3$ (Supplement Figure 1). The baseline data of the two groups were comparable which were showed in the Supplement Table 1.

With regard to the cutoff date, there were 15 long-term survivors who were disease-free for more than 60 months without treatment after obtaining a CR during the novel treatment. Among these 15 patients, 12 patients were still alive with no evidence of disease after treatment with a disease-free survival time from $60+$ to $135+$ months, as shown in Table 3 and Figure 2; two patients died of disease progression while in CR at 64 and 72 months after treatment; and one patient died of acute leukemia at 64 months after treatment.

AEs are shown in Table 4. During induction therapy, the most common AEs $\geq$ grade 3 were leucopenia (39.5\%), acne-like rash (11.6\%), febrile neutropenia (14\%), and thrombocytopenia (9.3\%). Frequent grade $3 / 4$ toxicities exceeded $10 \%$ of patients during CCRT, including oral mucositis (39.1\%), dermatitis (infield) $(26.1 \%)$, leukopenia (17.4\%), acne-like rash $(13 \%)$, and thrombocytopenia (13\%). Severe (i.e., grade 3/4) toxicities during maintenance treatment were rare, including hand-foot skin reactions in one patient and hyperbilirubinemia in one patient, and these 2 patients discontinued treatment because of the toxic effects. No patients died during treatment or within 30 days of completion of CCRT. Except for some acne-like rash in patients with the novel regimen but not in patients with conventional regimens, the novel regimen did not result in increased AEs according to the toxicities grade classification.

The median OS was unreached (95\% CI undefined; eight events) in patients with IM and was 20.3 months (95\% CI, 13.826.8 ; 23 events) in patients with RM (HR, 3.4; 95\% CI, 1.6-6.6, $p$ $=0.0013$; Figure 3A). In patients with IM, the median PFS was more than 44 months (eight events; [51.5\% of deaths were in 44

TABLE 3 | Characters and survival outcome of long-term disease-free survivors.

\begin{tabular}{|c|c|c|c|c|c|c|c|}
\hline Patient & Gender & Age & Group & Metastatic sites & EBV status & $\begin{array}{c}\text { Response of } \\
\text { introduction } \\
\text { treatment }\end{array}$ & $\begin{array}{c}\text { Disease-free survival } \\
\text { time (months) }\end{array}$ \\
\hline 1 & Male & 63 & $\mathrm{IM}$ & Bone & Negative & $\mathrm{CR}$ & 102 \\
\hline 2 & Female & 48 & $\mathrm{IM}$ & Bone, liver, lung & Positive & $\mathrm{CR}$ & 120 \\
\hline 3 & Female & 43 & $\mathrm{IM}$ & Lung & Negative & $\mathrm{CR}$ & 61 \\
\hline 4 & Female & 63 & $\mathrm{IM}$ & Bone, distant lymph node, pelvic & Positive & $\mathrm{CR}$ & 70 \\
\hline 5 & Female & 46 & $\mathrm{IM}$ & Bone & Negative & CR & 69 \\
\hline 6 & Male & 46 & $\mathrm{IM}$ & Bone & Positive & $\mathrm{CR}$ & 67 \\
\hline 7 & Male & 43 & $\mathrm{IM}$ & Bone & Negative & $\mathrm{CR}$ & 60 \\
\hline 8 & Male & 45 & $\mathrm{IM}$ & Bone & Negative & $P R$ & 74 \\
\hline 9 & Male & 23 & $\mathrm{RM}$ & Bone, lung & Positive & $\mathrm{CR}$ & 128 \\
\hline 10 & Male & 36 & $\mathrm{RM}$ & Bone, lung, pleura & Negative & $\mathrm{CR}$ & 101 \\
\hline 11 & Male & 43 & $\mathrm{RM}$ & Lung & Negative & $\mathrm{CR}$ & 97 \\
\hline 12 & Male & 40 & $\mathrm{IM}$ & Liver, lung & Negative & $\mathrm{CR}$ & 135 \\
\hline
\end{tabular}



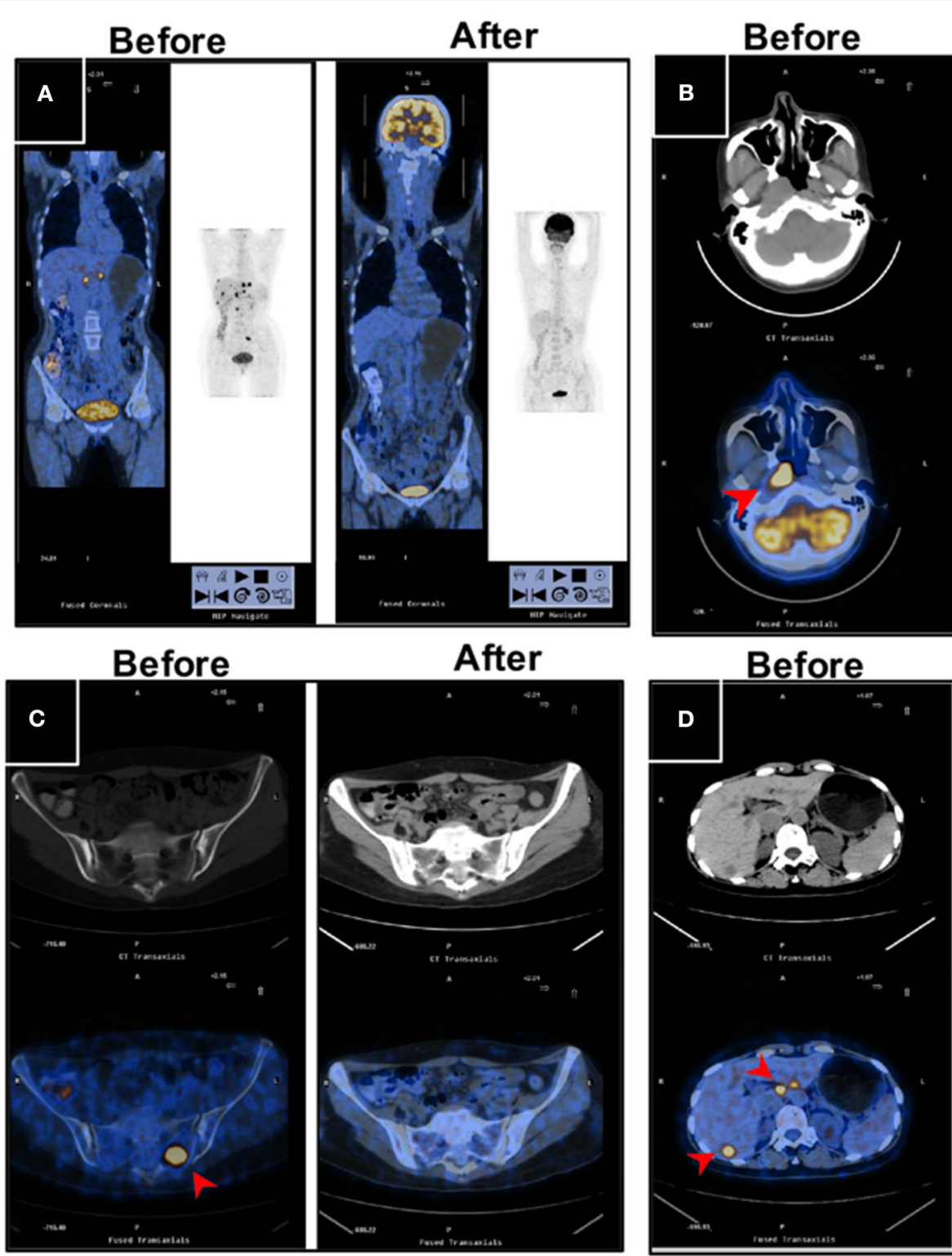

Before

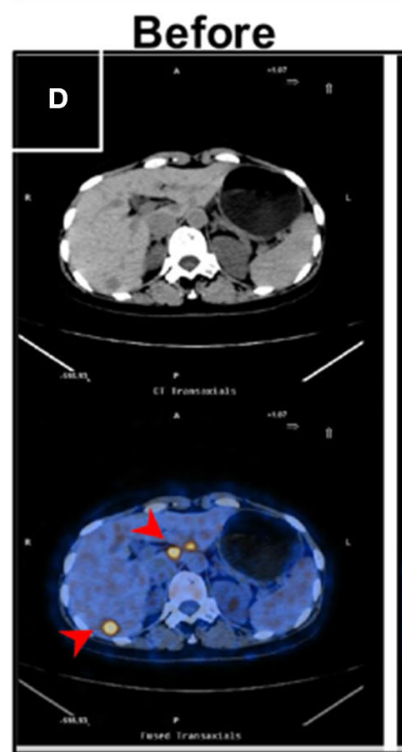

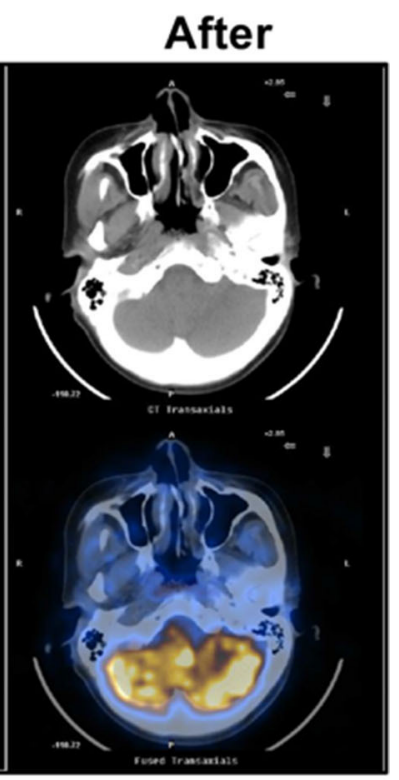

After

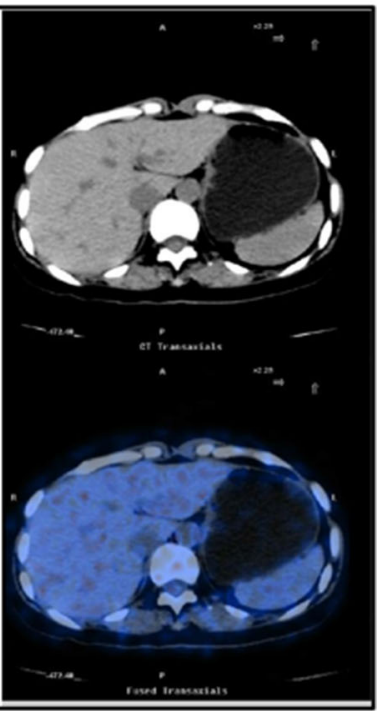

FIGURE 2 | PET/CT images for a long-term disease-free patient before and after the novel regimen. The female patient, 48 years of age, with an initial diagnosis of nasopharyngeal carcinoma with bone and liver metastases, EBV+, survived without disease for more than 120 months. (A) The systemic lesions, (B) the primary nasopharyngeal tumors, (C) the bone metastases, and (D) the liver metastases disappeared or decreased after treatment compared with before treatment.

mo]) vs. 12.5 months (95\% CI, 11.2-17.0; 23 events) in patients with RM (HR, 2.7, 95\% CI, 1.3-5.2; $p=0.009$; Figure 3B).

Post-hoc analysis showed that the IM group had a higher CR rate $(9 / 17,52.9 \%$; 95\% CI, 29.2-76.7\%) compared with $23.1 \%$ $(6 / 26$; $95 \% \mathrm{CI}, 6.9-39.3 \%)$ in RM patients $(p=0.045)$. Indeed, the 15 patients with a CR had a significant longer OS than these patients without a CR after induction chemotherapy (median OS, undefined vs. 20.3 months [95\% CI, 15-25.6], $p<0.001$ ), with a better OS at 2 years (93.3 vs. $42.9 \%)$ and 5 years $(82.2$ vs. $7.1 \%)$ and a lower risk of death (HR, 8.3, 95\% CI, 3.514.5, $p<0.000$; Figure 4A). Correspondingly, these patients also exhibited a better PFS (median PFS, undefined vs. 14.1 months
[95\% CI 11.8-16.4], HR 7.1, 95\% CI, 2.7-10.9, $p<0.0001)$ and a higher 2-year PFS (80 vs. $21.4 \%$ ) and 5-year PFS (80 vs. $7.1 \%$ ) (Figure 4B).

\section{DISCUSSION}

Despite advances in radiotherapy and effective systemic agents during the past decade, the long-term survival of patients with $\mathrm{mNPC}$ remains poor. The standard first-line treatment of platinum-containing doublet regimens for $\mathrm{mNPC}$ is essentially palliative therapy. This new therapeutic strategy in our study 
TABLE 4 | Adverse events during different periods of treatment in the study group.

\begin{tabular}{|c|c|c|c|c|c|c|}
\hline \multirow[b]{2}{*}{ Toxicity } & \multicolumn{2}{|c|}{ Induction $(N=43)$} & \multicolumn{2}{|c|}{ CCRT $(N=34)$} & \multicolumn{2}{|c|}{ Maintenance $(N=15)$} \\
\hline & Any grade & Grade $\geq 3$ & Any grade & Grade $\geq 3$ & Any grade & Grade $\geq 3$ \\
\hline Leukopenia & $31(72.1)$ & 17 (39.5) & $16(47.1)$ & $6(17.6)$ & $1(6.0)$ & 0 \\
\hline Acne-like rash & $19(44.2)$ & 5 (11.6) & $10(29.4)$ & $4(11.8)$ & $2(13.3)$ & 0 \\
\hline Dermatitis (in-field) & 0 & 0 & $20(58.8)$ & $8(23.5)$ & 0 & 0 \\
\hline Nausea & $18(41.9)$ & 0 & $6(17.6)$ & $3(8.8)$ & $2(13.3)$ & 0 \\
\hline Vomiting & $6(14.0)$ & 0 & $3(8.8)$ & $2(5.9)$ & $1(6.0)$ & 0 \\
\hline Oral mucositis & 8 (18.6) & 0 & $22(64.7)$ & $13(38.2)$ & $2(13.3)$ & 0 \\
\hline Febrile neutropenia & $6(14.0)$ & $6(14.0)$ & $4(11.8)$ & $3(8.8)$ & 0 & 0 \\
\hline Hyperbilirubinemia & $3(7.0)$ & 0 & $2(5.9)$ & 0 & $2(13.3)$ & $1(6.0)$ \\
\hline Infusion reaction & $3(7.0)$ & 0 & 0 & 0 & 0 & 0 \\
\hline Infection & $2(4.7)$ & 0 & $6(17.6)$ & $2(5.9)$ & 0 & 0 \\
\hline Diarrhea & $4(9.3)$ & 0 & $3(8.8)$ & 0 & $1(6.0)$ & 0 \\
\hline Premature heartbeat & $1(2.3)$ & 0 & $1(2.9)$ & 0 & 0 & 0 \\
\hline Alopecia & 10 (23.3) & 0 & $5(14.7)$ & $1(2.9)$ & 0 & 0 \\
\hline Thrombocytopenia & $5(11.6)$ & $4(9.3)$ & $6(17.6)$ & $4(11.8)$ & 0 & 0 \\
\hline Transaminitis & $2(4.7)$ & 0 & $4(11.8)$ & 0 & $1(6.0)$ & 0 \\
\hline Anemia & $3(7.0)$ & $1(2.3)$ & $6(17.6)$ & $3(8.8)$ & 0 & 0 \\
\hline Hypokalemia & $2(4.7)$ & 0 & $2(5.9)$ & $1(2.9)$ & $1(6.0)$ & 0 \\
\hline Peripheral neuropathy & 0 & 0 & $17(50.0)$ & $1(2.9)$ & $1(6.0)$ & 0 \\
\hline Hand-foot skin reaction & 0 & 0 & 0 & 0 & $4(26.7)$ & $1(6.0)$ \\
\hline Dysphagia & 0 & 0 & 12 (35.3\%) & 7 (20.6) & & \\
\hline
\end{tabular}

yielded significantly long durations of OS and PFS (5-year OS, 33.2\%; 5-year PFS, 29\%). Moreover, further subgroup analyses suggested that patients who were not pretreated with radiotherapy achieved better outcomes than radiotherapypretreated patients. The 5-year OS and PFS were 54.4 and 51.5\% in initially diagnosed $\mathrm{mNPC}$ patients, respectively. This finding may be associated with the history of radiotherapy. Previous ionizing radiation may increase chemotherapy resistance, as confirmed in prostate cancer and chronic myeloid leukemia $(19,20)$. A low survival rate in the contemporaneous controls was observed in our center (5-year OS, 10.9\%; 5-year PFS, 0\%), which was in accordance with previous reports. The favorable outcome of the novel regimen indicates the possible opportunity to completely cure chemotherapy-naïve mNPC, especially in patients with IM.

A long survival time is particularly prominent for patients who achieve a CR or PR of metastatic lesions after systemic chemotherapy (21). One study analyzed these different treatment combinations (induction, concurrent, and maintenance chemotherapy) and found that only induction-based chemotherapy was associated with significantly improved survival (22). In our study, the OR and CR rates after induction chemotherapy were 79.1 and $34.9 \%$, respectively. Furthermore, $94 \%$ of patients with IMs achieved objective remission, and more than half of them exhibited CR after induction chemotherapy. Induction therapy consisting of cetuximab plus cisplatin and docetaxel in the regimen conferred a significant improvement in the response rate, especially the $\mathrm{CR}$ rate, vs. historic controls (OR rate, $60-74 \%$; CR rate, 3-7\%) (9) and contemporaneous controls
(OR rate, 47\%; CR rate, 3\%) in our center. These results imply that adding cetuximab to induction chemotherapy improved chemotherapy outcomes. In fact, anti-EGFR monoclonal antibody therapy can improve the effect of chemotherapy or reverse resistance to the chemotherapy agent. Cetuximab was shown in a previous study to circumvent irinotecan resistance in irinotecan-refractory colorectal cancer (23). In metastatic/recurrent head and neck squamous-cell carcinoma (HNSCC) or squamous-cell lung cancer, the addition of these molecular-targeted agents, such as cetuximab, nimotuzumab, panitumumab, necitumumab, to platinum-based chemotherapy also improves the response rate and survival $(13,24-27)$. Chan et al. found a dose-dependent additive enhanced antitumor activity when cetuximab was combined with cisplatin or taxanes in NPC cell lines (28) and then confirmed its clinical activity in combination with carboplatin in heavily pretreated patients with mNPC (14).

Several studies have shown that radiotherapy to the primary tumor site combined with active systematic therapy can improve the survival of patients with stage $\operatorname{IVc} \operatorname{NPC}(29,30)$. AntiEGFR-targeted agents have been demonstrated to improve the effect of chemoradiotherapy or to reverse radiotherapy resistance $(12,31,32)$. The multicenter ENCORE study (33) and a phase 2 study (31) in Hong Kong Prince of Wales Hospital both showed prolonged 2-year PFS beyond 85\% compared with historic data in patients with locoregional advanced NPC who received cetuximab-added chemoradiotherapy. During our study, among 34 patients who attained an objective response after induction therapy and continued to receive CCRT, 33 


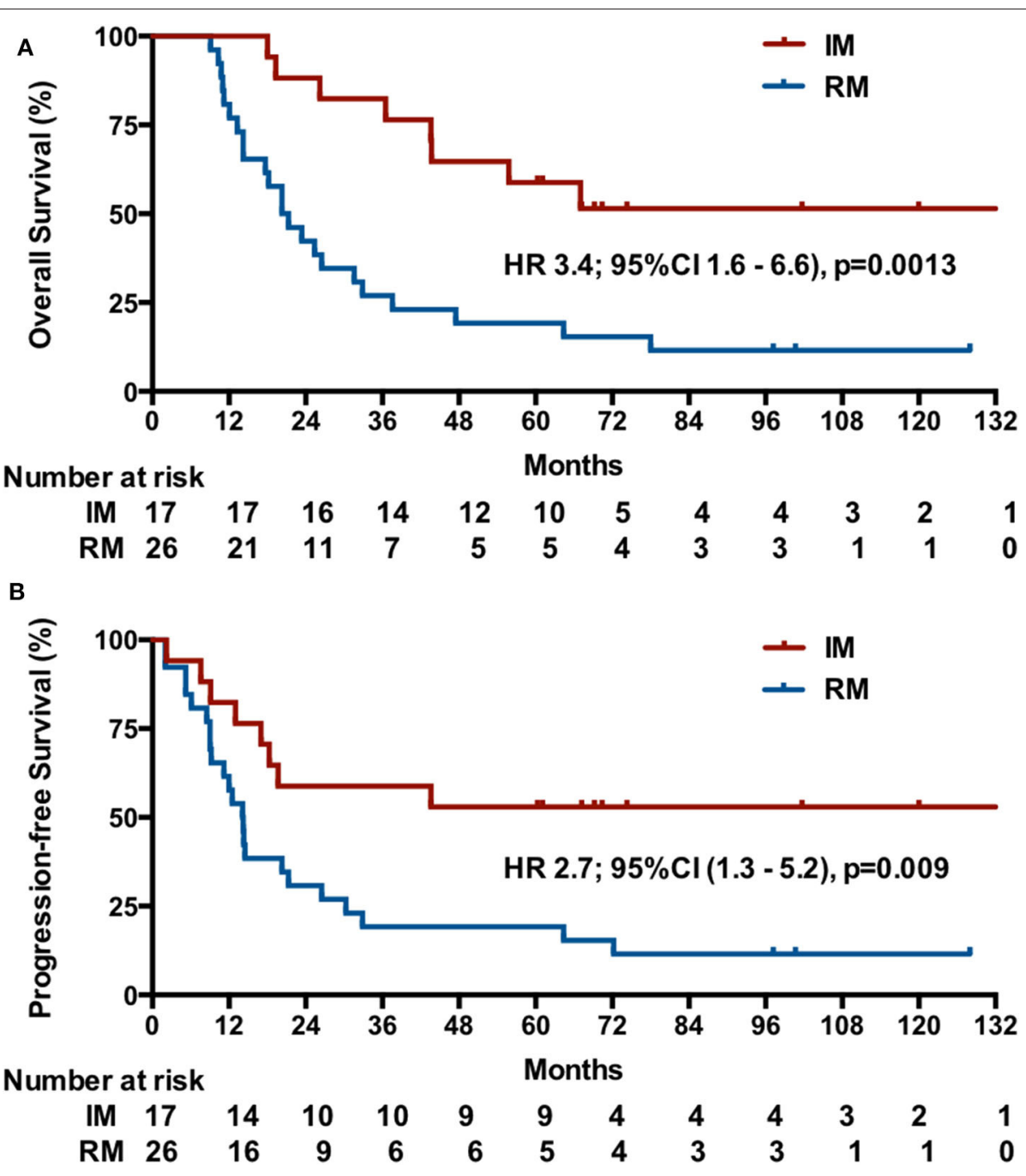

FIGURE 3 | Kaplan-Meier estimates of OS (A) and PFS (B) among patients initially diagnosed with mNPC (IM) or NPC patients with first-relapse metastases after radiotherapy $(\mathrm{RM})$.

achieved further remission, and one case exhibited PD. Another anti-EGFR humanized antibody, nimotuzumab, also provided survival benefit when used concurrently with chemoradiotherapy in $\operatorname{HNSCC}(34,35)$. Nevertheless, the addition of panitumumab to CCRT did not confer any benefit in HNSCC (36). The role of these EGFR antagonists in $\mathrm{MNPC}$ needs to be assessed in the future. The investigations in the studies above have demonstrated the safety and tolerability of cetuximab in patients with locoregionally advanced or recurrent and/or metastatic NPC. However, our study is the first to explore the addition of cetuximab to two processes of one regimen, i.e., induction and chemoradiation. There were few grade 3 skin reactions and no treatment-related mortalities or discontinuations of therapy reported during the entire treatment period. Importantly, in the last years local therapy of oligometastatic disease shows improvement of overall survival in several types of cancer. In our study patients also underwent local therapy of metastatic disease whenever possible. Therefore, not only systemic therapy but also local therapy may improve the overall survival. However, it required a further study to confirm the function of local therapy for residual metastatic foci after induction therapy.

In the present study we selected capecitabine but not cetuximab as maintenance therapy based on the following reasons: first, at present, fluorouracil or capecitabine plus cisplatin is one of the widely used regimens in patients with recurrent or metastatic NPC. Moreover, single-agent capecitabine as a maintenance treatment has already shown a favorable safety profile in other metastatic cancers $(37,38)$. Second, based on our clinical trial initiated by investigator rather than a company-sponsored study, it is difficult for most patients to afford the high cost of cetuximab for a long maintenance therapy. Last, capecitabine is more convenient for 


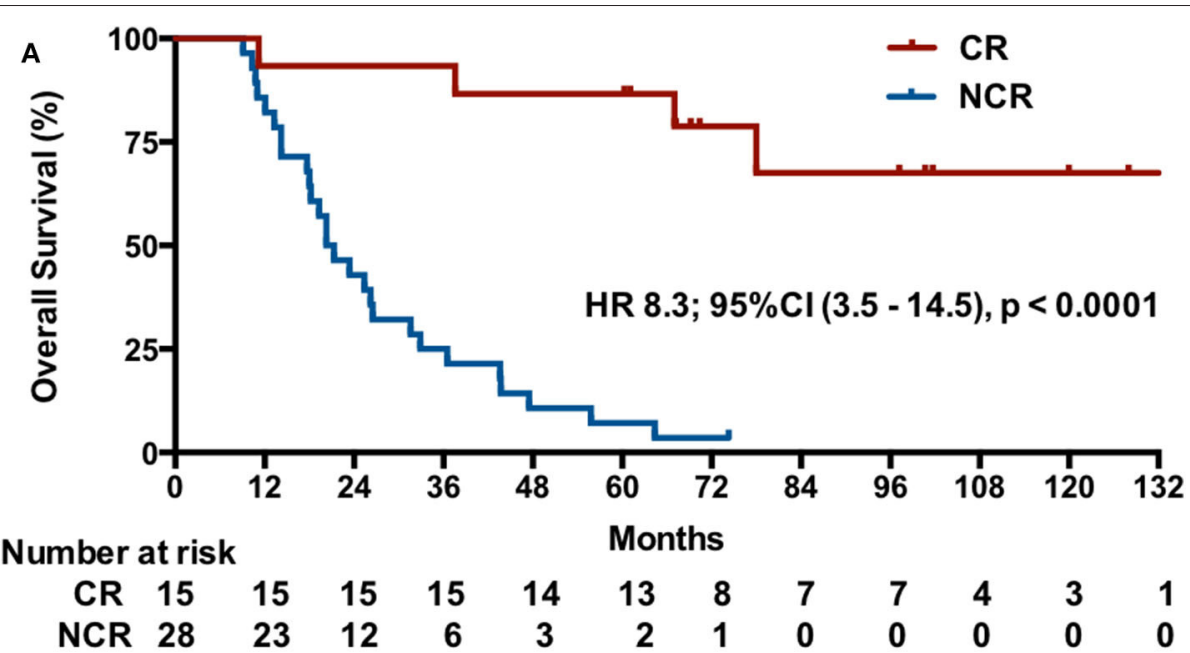

B
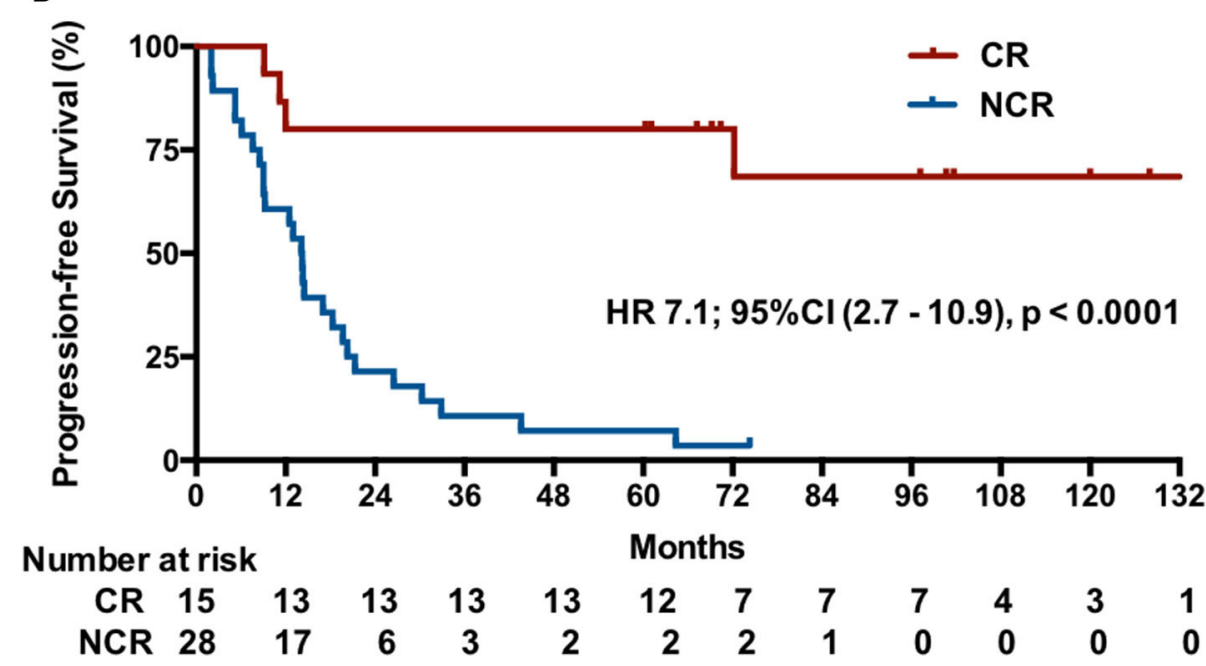

FIGURE 4 | Kaplan-Meier estimates of the OS (A) and PFS (B) by CR after induction chemotherapy.

oral administration, which does not require weekly intravenous injection like cetuximab. However, our data showed that onethird of patients had PD during the oral administration of capecitabine as maintenance treatment, suggesting the need for further exploration of the role of this strategy. In addition, anti-PD-1 antibodies $(39,40)$ have shown promising antitumor activity (OR rate $>20 \%$ ) for multiply pretreated $\mathrm{mNPC}$, which may be considered as another choice for maintenance therapy.

Our regimen was derived from this above evidence and showed good outcomes. Metastatic NPC appears to be incurable from the current literature. Few studies have reported the 5-year OS for mNPC, while patients with $\mathrm{mNPC}$ at initial diagnosis obtained a 54.4\% 5-year OS rate in our study. Although few longterm survivors after various aggressive treatments were presented in a retrospective study (4), currently, no prospective study has reported a definite regimen that could result in a considerable long-term survival rate for mNPC. In our study, 15 patients (34.9\%) who achieved long-term survival (>60 months), among whom, 12 were still alive with no evidence of disease at the 60 to 135 -month follow-ups. Our data suggest a potential curative role for chemotherapy-naïve $\mathrm{mNPC}$ when the novel regimen is applied. To the best of our knowledge, this study is the first report of a series of long-term survivors with mNPC. Although this study was a non-randomized and single-armed phase II study trial, we have to realize that the novel study regime at the time of 2006 is a very bold, new and high-intensity scheme with the attempt to achieve an expected long survival. Considering this limitation, we have currently initiated a randomized multicenter phase 3 trial (NCT02633176) in 2015 to further investigate this topic.

\section{DATA AVAILABILITY STATEMENT}

The datasets generated for this study are available on request to the corresponding author. 


\section{ETHICS STATEMENT}

The studies involving human participants were reviewed and approved by the ethics committee of Sun Yatsen University Cancer Center. The patients/participants provided their written informed consent to participate in this study.

\section{AUTHOR CONTRIBUTIONS}

TLi: conception and design. $\mathrm{HHu}, \mathrm{YH}, \mathrm{SL}$, and TLi: development of methodology. $\mathrm{MZ}, \mathrm{HHu}, \mathrm{XL}, \mathrm{YH}, \mathrm{CC}, \mathrm{XFa}, \mathrm{SL}$, and HHo: acquisition of data. MZ, HHu, XL, ZW, CG, SL, $\mathrm{XFu}, \mathrm{TLu}$, and TLi: analysis and interpretation of data. MZ, $\mathrm{TLi}, \mathrm{HHu}$, and $\mathrm{XL}$ : writing, review and/or revision of the manuscript. $\mathrm{HHu}, \mathrm{YT}$, and TLu: administrative, technical, or material support. TLi and TLu: study supervision. All authors contributed to the article and approved the submitted version.

\section{REFERENCES}

1. Ferlay J, Soerjomataram I, Dikshit R, Eser S, Mathers C, Rebelo M, et al. Cancer incidence and mortality worldwide: sources, methods and major patterns in GLOBOCAN 2012. Int J Cancer. (2015) 136:E359-86. doi: $10.1002 /$ ijc. 29210

2. Perez CA, Devineni VR, Marcial-Vega V, Marks JE, Simpson JR, Kucik N. Carcinoma of the nasopharynx: factors affecting prognosis. Int $J$ Radiat Oncol Biol Phys. (1992) 23:271-80. doi: 10.1016/0360-3016(92) 90741-Y

3. Tang L-Q, Chen Q-Y, Fan W, Liu H, Zhang L, Guo L, et al. Prospective study of tailoring whole-body dual-modality [18F]fluorodeoxyglucose positron emission tomography/computed tomography with plasma Epstein-Barr virus DNA for detecting distant metastasis in endemic nasopharyngeal carcinoma at initial staging. J Clin Oncol. (2013) 31:2861-9. doi: 10.1200/JCO.2012.46.0816

4. Fandi A, Bachouchi M, Azli N, Taamma A, Boussen H, Wibault P, et al. Long-term disease-free survivors in metastatic undifferentiated carcinoma of nasopharyngeal type. J Clin Oncol. (2000) 18:1324-30. doi: 10.1200/JCO.2000.18.6.1324

5. Chua DTT, Sham JST, Au GKH. A phase II study of docetaxel and cisplatin as first-line chemotherapy in patients with metastatic nasopharyngeal carcinoma. Oral Oncol. (2005) 41:589-95. doi: 10.1016/j.oraloncology.2005.01.008

6. Li YH, Wang FH, Jiang WQ, Xiang XJ, Deng YM, Hu GQ, et al. Phase II study of capecitabine and cisplatin combination as first-line chemotherapy in Chinese patients with metastatic nasopharyngeal carcinoma. Cancer Chemother Pharmacol. (2008) 62:539-44. doi: 10.1007/s00280-0070641-2

7. Zhang L, Huang Y, Hong S, Yang Y, Yu G, Jia J, et al. Gemcitabine plus cisplatin versus fluorouracil plus cisplatin in recurrent or metastatic nasopharyngeal carcinoma: a multicentre, randomised, open-label, phase 3 trial. Lancet. (2016) 388:1883-92. doi: 10.1016/S0140-6736(16)31388-5

8. Chua MLK, Wee JTS, Hui EP, Chan ATC. Nasopharyngeal carcinoma. Lancet. (2016) 387:1012-24. doi: 10.1016/S0140-6736(15)00055-0

9. Jin Y, Shi YX, Cai XY, Xia XY, Cai YC, Cao Y, et al. Comparison of five cisplatin-based regimens frequently used as the first-line protocols in metastatic nasopharyngeal carcinoma. J Cancer Res Clin Oncol. (2012) 138:1717-25. doi: 10.1007/s00432-012-1219-x

\section{FUNDING}

This study was supported by National Natural Science Foundation International (Regional) Cooperation and Exchange Project (81661168011).

\section{ACKNOWLEDGMENTS}

The authors thank all patients who participated in the study and their families; all medical staff who contributed to the study.

\section{SUPPLEMENTARY MATERIAL}

The Supplementary Material for this article can be found online at: https://www.frontiersin.org/articles/10.3389/fonc. 2020.01011/full\#supplementary-material

Supplement Figure 1 | Kaplan-Meier estimates of the OS (A) and PFS (B) among patients receving the novel regimen or conventional treatment.

Supplement Table 1 | Demographic and clinical characteristics of the patients receiving novel regimen and conventional regimen.
10. Kimura H, Sakai K, Arao T, Shimoyama T, Tamura T, Nishio K. Antibodydependent cellular cytotoxicity of cetuximab against tumor cells with wildtype or mutant epidermal growth factor receptor. Cancer Sci. (2007) 98:127580. doi: 10.1111/j.1349-7006.2007.00510.x

11. Ma BBY, Poon TCW, To KF, Zee B, Mo FKF, Chan CML, et al. Prognostic significance of tumor angiogenesis, Ki 67, p53 oncoprotein, epidermal growth factor receptor and HER2 receptor protein expression in undifferentiated nasopharyngeal carcinoma-a prospective study. Head Neck. (2003) 25:86472. doi: 10.1002/hed.10307

12. Bonner JA, Harari PM, Giralt J, Azarnia N, Shin DM, Cohen RB, et al. Radiotherapy plus cetuximab for squamous-cell carcinoma of the head and neck. N Engl J Med. (2006) 354:567-78. doi: 10.1056/NEJMoa053422

13. Vermorken JB, Mesia R, Rivera F, Remenar E, Kawecki A, Rottey S, et al. Platinum-based chemotherapy plus cetuximab in head and neck cancer. $N$ Engl J Med. (2008) 359:1116-27. doi: 10.1056/NEJMoa0802656

14. Chan ATC, Hsu M-M, Goh BC, Hui EP, Liu T-W, Millward MJ, et al. Multicenter, phase II study of cetuximab in combination with carboplatin in patients with recurrent or metastatic nasopharyngeal carcinoma. J Clin Oncol. (2005) 23:3568-76. doi: 10.1200/JCO.2005.02.147

15. Gennari A, Stockler M, Puntoni M, Sormani M, Nanni O, Amadori D, et al. Duration of chemotherapy for metastatic breast cancer: a systematic review and meta-analysis of randomized clinical trials. J Clin Oncol. (2011) 29:2144-9. doi: 10.1200/JCO.2010.31.5374

16. Lachin JM. Biostatistical Methods: The Assessment of Relative Risks. New York, NY: John Wiley \& Sons. (2000). doi: 10.1002/9780470317051

17. Ngan RKC, Yiu HHY, Lau WH, Yau S, Cheung FY, Chan TM, et al. Combination gemcitabine and cisplatin chemotherapy for metastatic or recurrent nasopharyngeal carcinoma: report of a phase II study. Ann Oncol. (2002) 13:1252-8. doi: 10.1093/annonc/mdf200

18. Shuster JJ. Median follow-up in clinical trials. J Clin Oncol. (1991) 9:191-2. doi: 10.1200/JCO.1991.9.1.191

19. Dmytrenko IV, Fedorenko VG, Shlyakhtychenko TY, Sholoyko VV, Lyubarets TF, Malinkina TV, et al. The efficiency of tyrosine kinase inhibitor therapy in patients with chronic myeloid leukemia exposed to ionizing radiation due to the Chornobyl nuclear power plant accident. Probl Radiac Med Radiobiol. (2014) 19:241-55.

20. Spratt DE, Evans MJ, Davis BJ, Doran MG, Lee MX, Shah N, et al. Androgen receptor upregulation mediates radioresistance after ionizing radiation. Cancer Res. (2015) 75:4688-96. doi: 10.1158/0008-5472.CAN-15-0892 
21. Zeng L, Tian YM, Huang Y, Sun XM, Wang FH, Deng XW, et al. Retrospective analysis of 234 nasopharyngeal carcinoma patients with distant metastasis at initial diagnosis: therapeutic approaches and prognostic factors. PLOS ONE. (2014) 9:e108070. doi: 10.1371/journal.pone.0108070

22. Chen MY, Jiang R, Guo L, Zou X, Liu Q, Sun R, et al. Locoregional radiotherapy in patients with distant metastases of nasopharyngeal carcinoma at diagnosis. Chin J Cancer. (2013) 32:604-13. doi: 10.5732/cjc.013.10148

23. Cunningham D, Humblet Y, Siena S, Khayat D, Bleiberg H, Santoro A, et al. Cetuximab monotherapy and cetuximab plus irinotecan in irinotecanrefractory metastatic colorectal cancer. N Engl J Med. (2004) 351:337-45. doi: 10.1056/NEJMoa033025

24. Meng J, Gu QP, Meng QF, Zhang J, Li ZP, Si YM, et al. Efficacy of nimotuzumab combined with docetaxel-cisplatin-fluorouracil regimen in treatment of advanced oral carcinoma. Cell Biochem Biophys. (2014) 68:181-4. doi: 10.1007/s12013-013-9686-5

25. Vermorken JB, Stöhlmacher-Williams J, Davidenko I, Licitra L, Winquist E, Villanueva C, et al. Cisplatin and fluorouracil with or without panitumumab in patients with recurrent or metastatic squamous-cell carcinoma of the head and neck (SPECTRUM): an open-label phase 3 randomised trial. Lancet Oncol. (2013) 14:697-710. doi: 10.1016/S1470-2045(13)70181-5

26. Thatcher N, Hirsch FR, Luft AV, Szczesna A, Ciuleanu TE, Dediu M, et al. Necitumumab plus gemcitabine and cisplatin versus gemcitabine and cisplatin alone as first-line therapy in patients with stage IV squamous nonsmall-cell lung cancer (SQUIRE): an open-label, randomised, controlled phase 3 trial. Lancet Oncol. (2015) 16:763-74. doi: 10.1016/S1470-2045(15)00021-2

27. Burtness B, Goldwasser MA, Flood W, Mattar B, Forastiere AA, Eastern Cooperative Oncology Group. Phase III randomized trial of cisplatin plus placebo compared with cisplatin plus cetuximab in metastatic/recurrent head and neck cancer: an eastern cooperative Oncology Group study. J Clin Oncol. (2005). 23:8646-54. doi: 10.1200/JCO.2005.02.4646

28. Sung FL, Poon TCW, Hui EP, Ma BBY, Liong E, To KF, et al. Antitumor effect and enhancement of cytotoxic drug activity by cetuximab in nasopharyngeal carcinoma cells. In Vivo. (2005) 19:237-45.

29. Yeh SA, Tang Y, Lui CC, Huang EY. Treatment outcomes of patients with AJCC stage IVC nasopharyngeal carcinoma: benefits of primary radiotherapy. JPN J Clin Oncol. (2006) 36:132-6. doi: 10.1093/jjco/hyi245

30. Lin S, Tham IWK, Pan J, Han L, Chen Q, Lu JJ. Combined high-dose radiation therapy and systemic chemotherapy improves survival in patients with newly diagnosed metastatic nasopharyngeal cancer. Am J Clin Oncol. (2012) 35:474-9. doi: 10.1097/COC.0b013e31821a9452

31. Ma BBY, Kam MKM, Leung SF, Hui EP, King AD, Chan SL, et al. A phase II study of concurrent cetuximab-cisplatin and intensitymodulated radiotherapy in locoregionally advanced nasopharyngeal carcinoma. Ann Oncol. (2012) 23:1287-92. doi: 10.1093/annonc/ mdr401

32. Bonner JA, Harari PM, Giralt J, Cohen RB, Jones CU, Sur RK, et al. Radiotherapy plus cetuximab for locoregionally advanced head and neck cancer: 5-year survival data from a phase 3 randomised trial, and relation between cetuximab-induced rash and survival. Lancet Oncol. (2010) 11:21-8. doi: 10.1016/S1470-2045(09)70311-0

33. Lin T, Zhao C, Gao L, Lang JY, Pan JJ, Hu CS, et al. 8558 POSTER an open, multicenter clinical study of cetuximab combined With intensity modulated radiotherapy plus concurrent chemotherapy in locally advanced nasopharyngeal carcinoma. Eur J Cancer. (2011). 47:S561. doi: 10.1016/S0959-8049(11)72200-6

34. Basavaraj C, Sierra P, Shivu J, Melarkode R, Montero E, Nair P. Nimotuzumab with chemoradiation confers a survival advantage in treatment-naïve head and neck tumors over expressing EGFR. Cancer Biol Ther. (2010) 10:673-81. doi: 10.4161/cbt.10.7.12793

35. Rodríguez MO, Rivero TC, del Castillo Bahi R, Muchuli CR, Bilbao MA, Vinageras EN, et al. Nimotuzumab plus radiotherapy for unresectable squamous-cell carcinoma of the head and neck. Cancer Biol Ther. (2010) 9:343-9. doi: 10.4161/cbt.9.5.10981

36. Mesía R, Henke M, Fortin A, Minn H, Yunes Ancona AC, Cmelak A, et al. Chemoradiotherapy with or without panitumumab in patients with unresected, locally advanced squamous-cell carcinoma of the head and neck (CONCERT-1): a randomised, controlled, open-label phase 2 trial. Lancet Oncol. (2015) 16:208-20. doi: 10.1016/S1470-2045(14)71198-2

37. Zhang Y, Sun M, Huang G, Yin L, Lai Q, Yang Y, et al. Maintenance of antiangiogenic and antitumor effects by orally active low-dose capecitabine for long-term cancer therapy. Proc Natl Acad Sci USA. (2017) 114:E5226-35. doi: $10.1073 /$ pnas.1705066114

38. Cremolini C, Moretto R, Masi G, Falcone A. Safety profile of capecitabine as maintenance treatment after induction with XELOX or FOLFOX in metastatic colorectal cancer patients. Ann Oncol. (2016) 27:1810. doi: 10.1093/annonc/mdw208

39. Hsu C, Lee SH, Ejadi S, Even C, Cohen RB, Le Tourneau C, et al. Safety and antitumor activity of pembrolizumab in patients with programmed deathligand 1-positive nasopharyngeal carcinoma: results of the KEYNOTE-028 study. J Clin Oncol. (2017) 35:4050-6. doi: 10.1200/JCO.2017.73.3675

40. Ma BBY, Lim W-T, Goh B-C, Hui EP, Lo K-W, Pettinger A, et al. Antitumor activity of nivolumab in recurrent and metastatic nasopharyngeal carcinoma: an international, multicenter study of the mayo clinic phase 2 consortium (NCI-9742). J Clin Oncol. (2018) 36:1412-8. doi: 10.1200/JCO.2017.77.0388

Conflict of Interest: The authors declare that the research was conducted in the absence of any commercial or financial relationships that could be construed as a potential conflict of interest.

Copyright (C) 2020 Zhang, Huang, Li, Huang, Chen, Fang, Wang, Guo, Lam, Fu, Hong, Tian, Lu and Lin. This is an open-access article distributed under the terms of the Creative Commons Attribution License (CC BY). The use, distribution or reproduction in other forums is permitted, provided the original author(s) and the copyright owner(s) are credited and that the original publication in this journal is cited, in accordance with accepted academic practice. No use, distribution or reproduction is permitted which does not comply with these terms. 